\section{PWE-124 THE DEVELOPMENT OF SQUAMO-COLUMNAR JUNCTION LOCATOR PROBE AND ITS PERFORMANCE ON BENCH STUDIES}

\author{
doi:10.1136/gut.2011.239301.387
}

of all studied orientations, an accuracy of less than $\pm 10 \mathrm{~mm}$ was achieved up to a distance of $15 \mathrm{~mm}$ between the magnet and the probe. Increase in temperature from $20^{\circ} \mathrm{C}$ to $37^{\circ} \mathrm{C}$ did not affect accuracy of the system even though it reduces the magnetic field strength. The manometer had a minor impact on the system's accuracy due to metallic properties of its sensors but no interference was seen with the $\mathrm{pH}$ probe.

Conclusion This technique should allow monitoring of the location of the SC junction to an accuracy greater than that of currently available high resolution manometry or multiple $\mathrm{pH}$ sensor devices.

Competing interests None.

Keywords squamo-columnar junction, Hall Effect, development, accuracy.

Y Y Lee, ${ }^{1, *}$ J G H Whiting, ${ }^{2}$ E V Robertson, ${ }^{1}$ J P Seenan, ${ }^{1}$ P Connolly, ${ }^{2}$ K E L McColl1 ${ }^{1}$ Institute of Cardiovascular and Medical Sciences, University of Glasgow, Glasgow, UK; ${ }^{2}$ Bioengineering, University of Strathclyde, Glasgow, UK

Introduction The position of the squamo-columnar (SC) junction is constantly changing and this precludes accurate monitoring of acid exposure of the most distal oesophagus. The authors have developed a novel probe to allow continuous monitoring of the SC junction location. The method involves endoscopically clipping a small magnet $(2 \mathrm{~mm}$ diameter and 1 $\mathrm{mm}$ thick) to the SC junction and monitoring its position relative to the probe comprising 26 Hall Effect sensors mounted at $5 \mathrm{~mm}$ spacing on a circuit board contained within a silastic tube (diameter $3.2 \mathrm{~mm}$ ). The aim of the current study was to assess the accuracy of the system on the bench.

Methods The accuracy was determined by comparing the recorded position of the magnet along the length of the probe with its actual position along the probe. This was assessed with the magnet in various rotations and orientations both anterior and posterior to the probe and various distances away from the probe. As the conductive property of Hall Effect sensors is dependent on temperature, bench studies were performed to assess effects of rising temperature on the system's accuracy. The potential electro-magnetic interference of the locator probe with multi-channels $\mathrm{pH}$ probe (Synectics, UK) and solid state high resolution manometer (Synmed, UK) was also investigated.

Results The accuracy was related to the distance between magnet and probe, orientation of the field of the magnet relative to the probe and whether the magnet was positioned anterior, posterior or lateral to the probe. Greatest accuracy was with the magnet anterior to the probe and its magnetic field perpendicular to the length of probe with position errors of $\pm 2.3, \pm 2.9, \pm 2.7 \mathrm{~mm}$ at 5,10 and $15 \mathrm{~mm}$ distance from the probe respectively. The poorest accuracy was with the magnet lateral to the probe and its magnetic field horizontal to the line of the probe, with position errors of $\pm 2.3, \pm 7.1, \pm 7.9 \mathrm{~mm}$ at 5 , 10 and $15 \mathrm{~mm}$ distance from the probe respectively. Over $95 \%$ 